\title{
Molecular and functional characterization of human bone marrow adipocytes
}

\author{
Antonella Poloni $^{\mathrm{a}}$, Giulia Maurizi ${ }^{\mathrm{a}}$, Federica Serrani $^{\mathrm{a}}$, Stefania Mancini ${ }^{\mathrm{a}}$, Maria Cristina Zingaretti ${ }^{\mathrm{b}}$, \\ Andrea Frontini ${ }^{\mathrm{b}}$, Saverio Cinti ${ }^{\mathrm{b}}$, Attilio Olivieri ${ }^{\mathrm{a}}$, and Pietro Leoni ${ }^{\mathrm{a}}$ \\ ${ }^{a}$ Clinica di Ematologia, Dipartimento Scienze Cliniche e Molecolari, Università Politecnica delle Marche-Azienda Ospedali Riuniti, Ancona, Italy; \\ ${ }^{\mathrm{b}}$ Dipartimento di Medicina Sperimentale e Clinica, Università Politecnica delle Marche-Azienda Ospedali Riuniti, Ancona, Italy
}

(Received 12 December 2012; revised 30 January 2013; accepted 3 February 2013)

\begin{abstract}
Adipocytes are a cell population largely located in the human bone marrow cavity. In this specific microenvironment where adipocytes can interact with a variety of different cells, the role of fat is mainly unknown. To our knowledge, this report is the first to characterize mature adipocytes isolated from human bone marrow (BM-A) molecularly and functionally to better understand their roles into the hematopoietic microenvironment. Healthy BM-A were isolated after collagenase digestion and filtration. We studied the morphology of BM$A$, their gene expression and immunophenotypic profile and their functional ability in the hematopoietic microenvironment, comparing them with adipocytes derived from adipose tissue (AT-A). BM-A showed a unilocular lipid morphology similar to AT-A and did not lose their morphology in culture; they showed a comparable pattern of stem cell-surface antigens to AT-A. In line with these observations, molecular data showed that BM-A expressed some embryonic stem cells genes, such as Oct4, KLf4, c-myc, Gata4, Tbx1, and Sox17, whereas they did not express the stem cell markers Sox 2 and Nanog. Moreover, BM-A had long telomeres that were similar to bone marrow mesenchymal stem cells. Notably, BM-A supported the survival and differentiation of hematopoietic stem cells in long-term cultures. These results showed that BM-A are stromal cells with a gene expression pattern that distinguished them from AT-A. BM-A showed stem cell properties through their hematopoietic supporting function, which was certainly linked to their role in the maintenance of the bone marrow microenvironment. Depending on specific demands, BM-A may acquire different functions based on their local environment. (C) 2013 ISEH - Society for Hematology and Stem Cells. Published by Elsevier Inc.
\end{abstract}

The bone marrow (BM) microenvironment contains many different types of cells. Hematopoietic and stromal stem cells are more highly studied than BM fat, which is an abundant component in adult BM [1]. Many studies have reported that active hematopoietic $\mathrm{BM}$, called red bone marrow, declines with age and is gradually converted to fatty marrow, called yellow bone marrow, from the periphery toward the axial skeleton [2]. With age, nonhematopoietic fatty marrow progressively predominates and fills the entire marrow cavity through a dynamic and reversible process [3].

Offprint requests to: Antonella Poloni, Ph.D., M.D., Clinica di Ematologia, Dipartimento di Scienze Cliniche e Molecolari, Università Politecnica delle Marche, Via Tronto 60020, Ancona, Italy; E-mail: a.poloni@ univpm.it

Supplementary data related to this article can be found online at http:// dx.doi.org/10.1016/j.exphem.2013.02.005.
Adipocytes represent a terminally differentiated cell population with a specific morphology characterized by the presence of a single, large cytoplasmic lipid droplet that accounts for approximately $90 \%$ of the cellular volume [4]. The role of fat in the BM microenvironment is largely unknown, and these cells have not been well characterized for their molecular and functional properties, contrary to adipose tissue adipocytes (AT-A) [5,6]. Recently, we demonstrated that mature human adipocytes isolated from the omental and subcutaneous adipose tissue depots have stem cell-like properties that could explain their plasticity and functional features [5-8].

We studied bone marrow adipocytes (BM-A) to try to understand their functions in the hematopoietic microenvironment and to compare their stemness with AT-A. Accordingly, we isolated and cultured BM-A in vitro and characterized these cells for their morphologic, molecular, and immunophenotypic properties. This study is the first 
to provide a detailed characterization of BM-A, studying the gene expression profile of genes involved in white and brown adipogenic lineage, stemness and lineage reprogramming, telomere length, and hematopoietic supporting capabilities. In addition to hematologic effects, information on marrow fat is of potential relevance to understand the fat plasticity. Indeed, this phenomenon is not limited to the adipose tissue, but could be demonstrated also in many fat-rich organs, such as parotid glands, parathyroid glands, thymus, lymph nodes, and the pancreas.

\section{Methods}

\section{Isolation and culture of mature adipocytes}

Bone marrow was harvested from the iliac crest of healthy BM donors ( $n=9$; median age, 45 years; range, 29-55 years) after obtaining written informed consent and in accordance with the guidelines of the local ethical committee (300-DG). Bone marrow $(5-10 \mathrm{~mL})$ from each donor was diluted with PBS and layered onto Ficoll (MP Biomedicals, Iukirch, France). The Ficoll gradient was spun at $370 \mathrm{~g}$ for $30 \mathrm{~min}$ at $4^{\circ} \mathrm{C}$. The floating adipocytes and the interphase mononuclear cells were collected. Subcutaneous fat tissues (5-10 g) were harvested from patients undergoing abdominal plastic surgery ( $\mathrm{n}=10$; median age, 49 years; range, 35-60 years) after obtaining written informed consent and in accordance with the guidelines of the local ethical committee (300-DG). After Ficoll separation, the BM samples were treated with a solution of $1 \mathrm{mg} / \mathrm{mL}$ of type I collagenase (Gibco; Invitrogen, Milan, Italy) and $2 \%$ human albumin at $37^{\circ} \mathrm{C}$ for 1 hour, whereas adipose tissue samples were minced into smaller pieces and incubated with $3 \mathrm{mg} / \mathrm{mL}$ of type I collagenase and $2 \%$ human albumin at $37^{\circ} \mathrm{C}$ for 2 hours. After digestion, the samples were filtered through a $200-\mu \mathrm{m}$ nylon sieve to obtain mature adipocytes that were free of other cells. The filtered cells were washed four times with Dulbecco's modified Eagle's medium (DMEM; Biological Industries, Milan, Italy) and centrifuged at $250 \mathrm{rcf}$ for $5 \mathrm{~min}$. Only the floating cell fraction was collected after each centrifugation step. Cells were seeded for ceiling culture as previously described [7,9] with DMEM supplemented with $20 \%$ fetal bovine serum (Stem Cell Technologies, Vancouver, $\mathrm{BC}, \mathrm{Canada}$ ) and cultured at $37^{\circ} \mathrm{C}$ and $5 \%$ $\mathrm{CO}_{2}$ or collected for molecular assays.

\section{Immunofluorescence of isolated adipocytes}

Confocal microscopy optical sectioning and computer-assisted image reconstruction of isolated adipocytes allowed us to exclude the presence of small, undifferentiated cells attached to the surface of the analyzed adipocytes. Isolated adipocytes were fixed in $4 \%$ paraformaldehyde, incubated with rabbit anti-perilipin (provided by Dr. A.S. Greenberg, Tufts University, Medford, MA, USA; 1:50 in PBS) and stained with FITC-linked secondary antibodies (Jackson ImmunoResearch, West Grove, PA, USA; 1:100 in PBS). Images were taken in the green channel. TOTO3 iodide (Molecular Probes, Invitrogen, Monza, Italy; 1:5000 in PBS) was used as the nuclear counterstain and visualized in the blue channel. We analyzed at least 100 adipocytes from each suspension. Images were sequentially obtained from two channels using a pinhole of 1:1200.

\section{Electron microscopy}

Bone marrow biopsy specimens collected for diagnostic reasons at electron microscopy service of our hospital were selected on the base of their adipocyte content. Solid small fragments of BM were immediately fixed in $2 \%$ glutaraldehyde $-2 \%$ formaldehyde in $0.1 \mathrm{~mol} / \mathrm{L}$ phosphate buffer $(\mathrm{pH} 7.4)$ for at least 4 hours, postfixed in a solution of $1 \%$ osmium-tetroxide, dehydrated in acetone, and finally embedded in epoxy resin. Significant areas from semithin resin sections $(1.5 \mu \mathrm{m})$ were selected to be studied at electron microscopy level; thin sections $(\sim 60 \mathrm{~nm})$ obtained with an MT-X Ultratome (RCM, Tucson, AZ, USA) were mounted on copper grids, stained with lead citrate, and examined with a CM10 transmission electron microscope (Philips, Eindhoven, The Netherlands).

\section{Immunophenotype analysis}

All BM-A and AT-A samples were characterized by flow cytometry after isolation. Cells were stained with fluorescein isothiocyanate (FITC)-, phycoerythrin (PE)-, or peridin chlorophyll protein (PerCP)-conjugated antibodies against CD90 (Thy-1; BD Pharmingen, San Diego, CA, USA), CD105 (endoglin; BD Pharmingen), CD271 (NGFR; Miltenyi Biotech, Cologne, Germany), CD117 (c-kit; Miltenyi Biotech), CD34 (hematopoietic progenitor cell antigen; BD Biosciences, Franklin Lakes, NJ), CD133 (hematopoietic stem cell antigen; Miltenyi Biotech), CD45 (leukocyte common antigen; BD Biosciences), and CD31 (platelet endothelial cell adhesion molecule; BD Biosciences). FITC-, PE- (Dako, Glostrup, Denmark), and PerCP- (BD Pharmingen) negative isotypes were used as control antibodies. Cells were incubated with primary antibodies at $4^{\circ} \mathrm{C}$ for $30 \mathrm{~min}$. Thereafter, cell fluorescence was evaluated using a FACSCalibur flow cytometer (Becton Dickinson). The data were analyzed using CellQuest software.

\section{Adipogenic lineage gene expression profile}

Total RNA was extracted using the RNeasy Plus Micro Kit (Qiagen, Milan, Italy) according to the manufacturer's instructions. The purity of the RNA was confirmed by determining the $260 \mathrm{~nm} / 280$ nm absorbance ratio ( $>1.8$ ). For each sample, $1 \mu \mathrm{g}$ of total RNA was reverse transcribed as described previously [7]. To quantify adipocyte lineage gene expression of adiponectin (NM_004797.2; $173 \mathrm{bp}$ ), fatty acid binding protein four (aP2, NM_001442.2; 133 $\mathrm{bp}$ ), peroxisome proliferators-activated receptor gamma coactivator 1 alpha (PGC1 alpha, NM_013261.3; 164 bp), leptin (LEP, NM_000230.2; 150 bp), cell death-inducing DFFA-like effector a (CIDEA, NM_001279.3; 167 bp), deiodinase type II (DIO2, NM-013989.4; 196 bp), real-time polymerase chain reaction (PCR) assays were performed with BM-A and AT-A samples. The transcript levels of these genes were normalized to the expression of the constitutive gene IPO8 (NM_006390.3; 185 bp) and an endogenous calibrator (BM-derived mesenchymal stem cells [BM-MSCs]) following the $2^{-\Delta \Delta C t}$ method [10]. The SYBR Select Master Mix (Life Technologies, Monza, Italy) and specific primers were optimized for the ABI 7500 Fast system (Applied Biosystems, Foster City, CA, USA) in a $20 \mu \mathrm{L}$ mixture, containing $10 \mu \mathrm{L}$ of MasterMix, $10 \mu \mathrm{mol} / \mathrm{L}$ of each primer and $2 \mu \mathrm{L}$ of cDNA template. After $20 \mathrm{sec}$ at $50^{\circ} \mathrm{C}$ and $10 \mathrm{~min}$ at $95^{\circ} \mathrm{C}, 40$ cycles of denaturation, $15 \mathrm{sec}$ at $95^{\circ} \mathrm{C}$, and annealing/extension for $1 \mathrm{~min}$ at $60^{\circ} \mathrm{C}$ were run. The MeltCurve Stage was performed using one cycle at $95^{\circ} \mathrm{C}$ for 15 $\mathrm{sec}, 60^{\circ} \mathrm{C}$ for $1 \mathrm{~min}, 95^{\circ} \mathrm{C}$ for $30 \mathrm{sec}$, and $60^{\circ} \mathrm{C}$ for $15 \mathrm{sec}$. Reverse transcriptase PCR assays were performed to quantify the expression 
of the following white and brown adipogenic-specific genes in BMA and AT-A: peroxisome proliferator-activated receptor gamma (PPAR $\gamma$, NM_138712.3, 225 bp), glycerol-3-phosphate dehydrogenase (GPDH, NM_005276.2; 215 bp), lipoprotein lipase (LPL, NM_000237.2; 221 bp), uncoupling protein 1 (UCP1, NM_021833.4; 199 bp) and PR domain containing 16 (PRDM16, NM_022114.3; 158 bp). Sense and antisense gene-specific primers were designed using the Primer3 software (http://frodo.wi.mit.edu/ primer3/), and primer pairs were designed to span at least one exonintron junction to exclude any possibility of genomic DNA amplification. PCR conditions used for these assays were previously described [7]. PCR products were separated by electrophoresis in a $2 \%$ agarose gel stained with ethidium bromide and visualized using an UV illuminator.

\section{Stem cells and cell reprogramming gene expression profile}

To quantify the gene expression level of the four specific cell reprogramming genes, octamer- binding transcription factor 4 (Oct4, NM_002701.4; 125 bp), Kruppel-like factor 4 (Klf4, NM_ 004235.4; 169 bp), oncogene myc (c-myc, NM_002467.4;248 bp) and (sex determining region Y)-box 2 (Sox2, NM_003106.3;191 bp), real-time PCR assays were performed with BM-A and ATA samples. The transcript levels of the genes tested were normalized to the expression of the constitutive gene IPO8 (NM_006390.3; 185 bp) and an endogenous calibrator (BMMSCs) following the $2^{-\Delta \Delta C t}$ method [9]. The ABI 7500 Fast 8 system (Applied Biosystems), and specific primers were used as described earlier. Reverse transcriptase PCR assays were performed to characterize BM-A and AT-A for the following stem cell-specific genes that are required for self-renewal and pluripotency: Gata4 (NM_002052.3;194 bp), T box 1 (Tbx1; NM_005992.1; 213 bp) sex determining region Ybox 17 (Sox17, NM_022454.3; 154 bp) and Nanog (NM_024865.2; 213 bp). Primers were designed using Primer3 software (http://frodo. wi.mit.edu/primer3/), and the PCR conditions used for these assays were described previously [6]. PCR products were separated by electrophoresis in $2 \%$ agarose gel stained with ethidium bromide and visualized using an UV illuminator.

\section{Telomere length analysis}

Telomere lengths of BM-A and AT-A were analyzed with a telomere restriction fragment length assay using the TeloTTAGGG telomere length assay (Roche, Mannheim, Germany). Genomic DNA was extracted using a QIAamp DNA Blood Mini kit (Qiagen). DNA concentration and purity were determined by spectrophotometry and gel electrophoresis. The assay was performed according to the manufacturer's protocol as described previously [11]. Data were obtained with a Chemidoc imaging system (Bio-Rad, Segrate, Italy) and analyzed using the Quantity One Software (Bio-Rad).

\section{Long-term culture-initiating cells}

Long-term culture-initiating cells (LTC-ICs) were used on three samples of BM-A, three samples of AT-A, and three samples of AT-derived MSCs, which were used as a positive control group, in 24-well plates. In accordance with the guidelines of the local ethics committee (300-DG), hematopoietic $\mathrm{CD}^{+}{ }^{+}$stem cells were isolated from healthy $\mathrm{BM}$ donors by immunomagnetic separation $\left(\mathrm{CD} 4^{+}\right.$Progenitor Cell Isolation Kit; Miltenyi Biotech). Next, $500 \mu \mathrm{L}$ of adipocytes and $5 \times 10^{4}$ cells/well of irradiated
AT-derived MSCs (80 Gy) were seeded in a final volume of 1 $\mathrm{mL}$ of the MyeloCult H5100 medium (StemCell Technologies) supplemented with $1 \%$ hydrocortisone. $\mathrm{CD} 34^{+}$cells were seeded

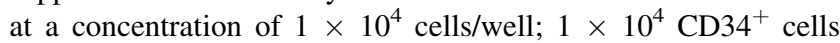
alone were seeded similarly to the negative control group. Cultures were performed in quadruplicate and incubated at $33^{\circ} \mathrm{C}$ in a fully humidified atmosphere with $5 \% \mathrm{CO}_{2}$. Cultures were fed every week by a half-medium change. The total progenitor cell content of each well was harvested after 8 weeks and plated in methylcellulose in $35 \mathrm{~mm}$ tissue culture dishes (Falcon). Assays for myeloid and erythroid progenitors (colony-forming unit [CFU] granulocyte macrophage [GM], and burst-forming unit-erythroid [BFU-E]) were performed in the methylcellulose medium HSCCFU Lite (Miltenyi Biotech) containing stem cell factor, interleukin (IL)-3, granulocyte-macrophage colony-stimulating factor (GM-CSF) and erythropoietin. The cultures were incubated at $37^{\circ} \mathrm{C}$ in a fully humidified atmosphere with $5 \% \mathrm{CO}_{2}$ for 14 days

\section{Quantitative cytokines assay}

After 7 days, the culture supernatants of adipocytes were collected and frozen at $-20^{\circ} \mathrm{C}$. Multiplex human cytokine, chemokine, and growth factor detection (BioPlex; Bio-Rad) were used to measure the production of IL-6, IL-8, IL-10, IL-17, granulocyte colonystimulating factor (G-CSF), granulocyte-macrophage colony-stimulating factor (GM-CSF), macrophage inflammatory protein (MIP)- $1 \alpha$, tumor necrosis factor- $\alpha$ (TNF- $\alpha)$ in the culture supernatants.

\section{Statistical analysis}

The data were presented as means \pm SD and analyzed with Student $t$ test. The differences were considered to be statistically significant for $p<0.05$.

\section{Results}

\section{Morphologic and immunophenotype analysis}

Light and electron microscopy of adipocytes corresponded to that described for adipocytes in other anatomical sites (i.e., with a thin cytoplasm with organelles and a large unilocular cytoplasmic lipid droplet that accounts for approximately $90 \%$ of the cellular volume and a small flattened nucleus; Fig. 1). We isolated a population of mature adipocytes from BM. Preliminary studies with confocal microscopy excluded any contamination by other cell types (data not shown); therefore, we isolated an homogeneous population of bona fide mature adipocytes. Primary cultures of BM-A exhibited a typical white adipocytic morphology corresponding to that described earlier. In vitro, BM-A maintained their shape without noticeable lipolysis, even after 30 days of culture time. Almost all the cells were unilocular adipocytes, and only a few cells seemed to take a more elongated morphology without losing their lipid droplets (Fig. 2). In vitro, AT-A spontaneously changed toward a fibroblast-like morphology characterized by the loss of their lipid droplets and the centralization of their nuclei after 6-10 days of culture. Next, the cells entered a proliferative expansion phase 

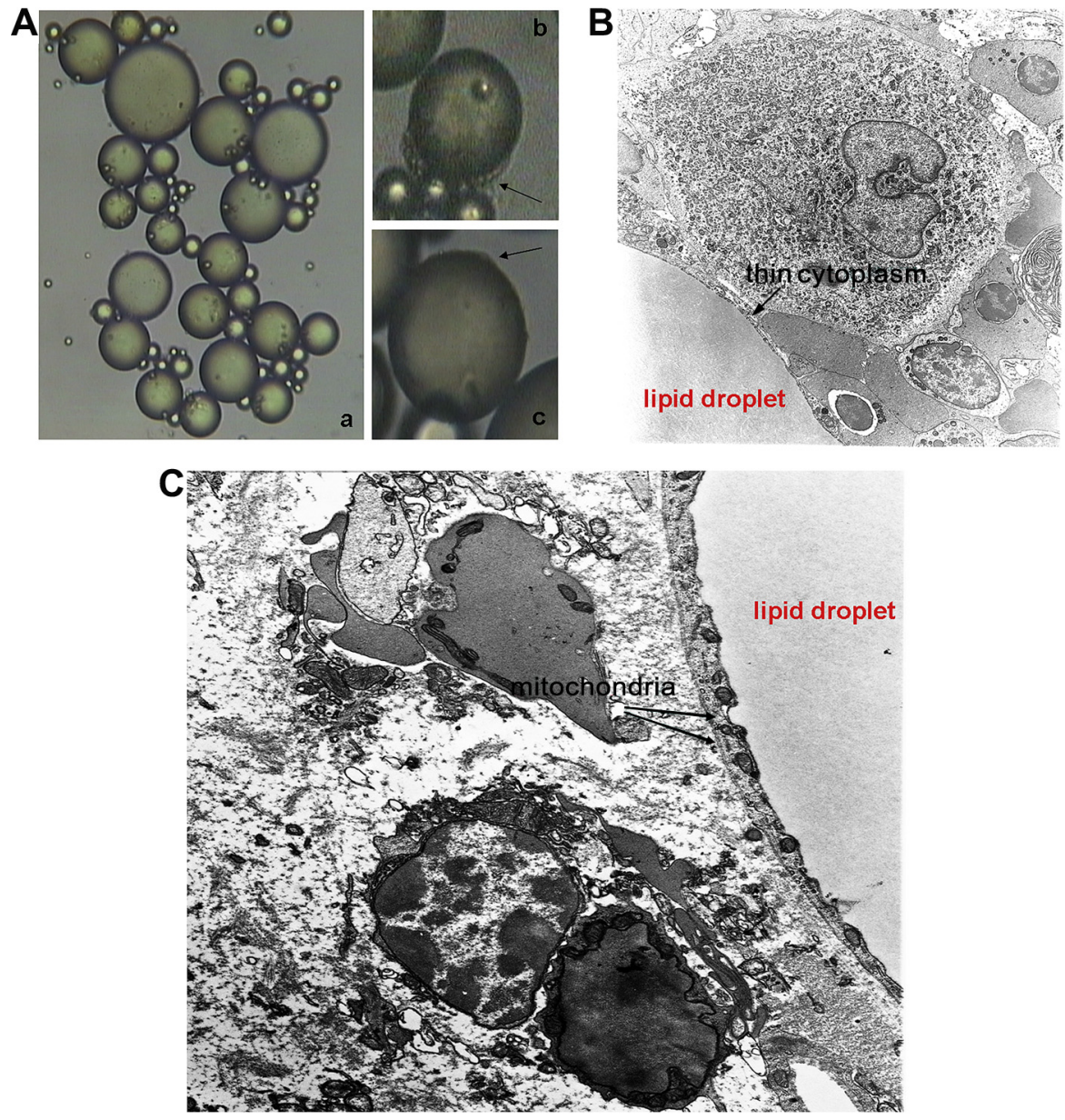

Figure 1. Microscopy analysis of BM-A. (A) Light microscopy analysis of BM-A in liquid culture. A thin cytoplasm of BM-A was observable in $(b)$ and (c). (a) Original magnification $\times 100$; scale bar $=70 \mu \mathrm{m}$. $(b, c)$ Original magnification $\times 200$. (B) Electron microscopy analysis of BM-A. Scale bar $=4 \mu \mathrm{m}$. (C) Scale bar $=2 \mu \mathrm{m}$.

until they reached cellular senescence. BM-A were analyzed for the expression of hematopoietic and mesenchymal stem cells markers, and the expression levels of these genes were compared with those of the AT-A. A similar pattern of surface antigen expression was observed in both cell types with some markers that were expressed at lower levels in BM-A compared with AT-A. Bone marrow fat was positively stained by CD90 $(7 \pm 3$ vs. $29 \pm 10$ in AT-A; $p<0.05)$, CD105 (35 \pm 10 vs. $39 \pm 11$ in AT-A), CD271 $(21 \pm 11$ vs. $70 \pm 0.3$ in AT-A; $p<0.05$ ), CD117 (6 \pm 3 vs. $17 \pm 9$ in ATA), CD34 (0.7 \pm 0.3 vs. $7 \pm 5$ in AT-A; $p<0.05)$, CD133 $(7 \pm 2$ vs. $7 \pm 1$ in AT-A) and CD31 ( $8 \pm 2$ vs. $17 \pm$ 9 in AT-A), while BM-A were not stained by CD45 (Supplementary Figure E1, online only, available at www.exphem.org). These data suggest that BM-A expressed typical hematopoietic and mesenchymal stem cells antigens, but CD34 was only present in a low percentage. Therefore, in this small fraction of cells with positive expression of hematopoietic stem cells this marker does not confer similar functional properties.

\section{Adipogenic gene expression profile}

To examine the lineage gene expression profile of BM-A, real-time PCR and reverse-transcriptase PCR assays were used to analyze the expression of mature adipocytespecific genes to determine whether BM-A have properties of both brown and white adipocytes. Data from BM fat displayed the expression of many white adipogenic genes. Adipocyte differentiation involves a series of transcriptional events, and PPAR $\gamma$, which was well expressed by $\mathrm{BM}$ fat samples, is one of the key positive transcriptional regulators of adipogenesis. Results showed the positive activation of the PPAR $\gamma$ target genes GPDH, LPL, Adiponectin, aP2 and LEP. Quantitative data showed a significantly lower expression of Adiponectin, aP2, and LEP in BM-A compared with AT-A. Moreover, Dio2, CIDEA, and PGC1- $\alpha$, regulators of brown adipocytes, were expressed, but there was no expression of the thermogenic marker UCP1 and the transcriptional regulator Prdm16. Comparative analysis between BM-A and AT-A showed a lower expression of CIDEA and PGC1- $\alpha$, and a higher expression of Dio2, respectively (Fig. 3). 

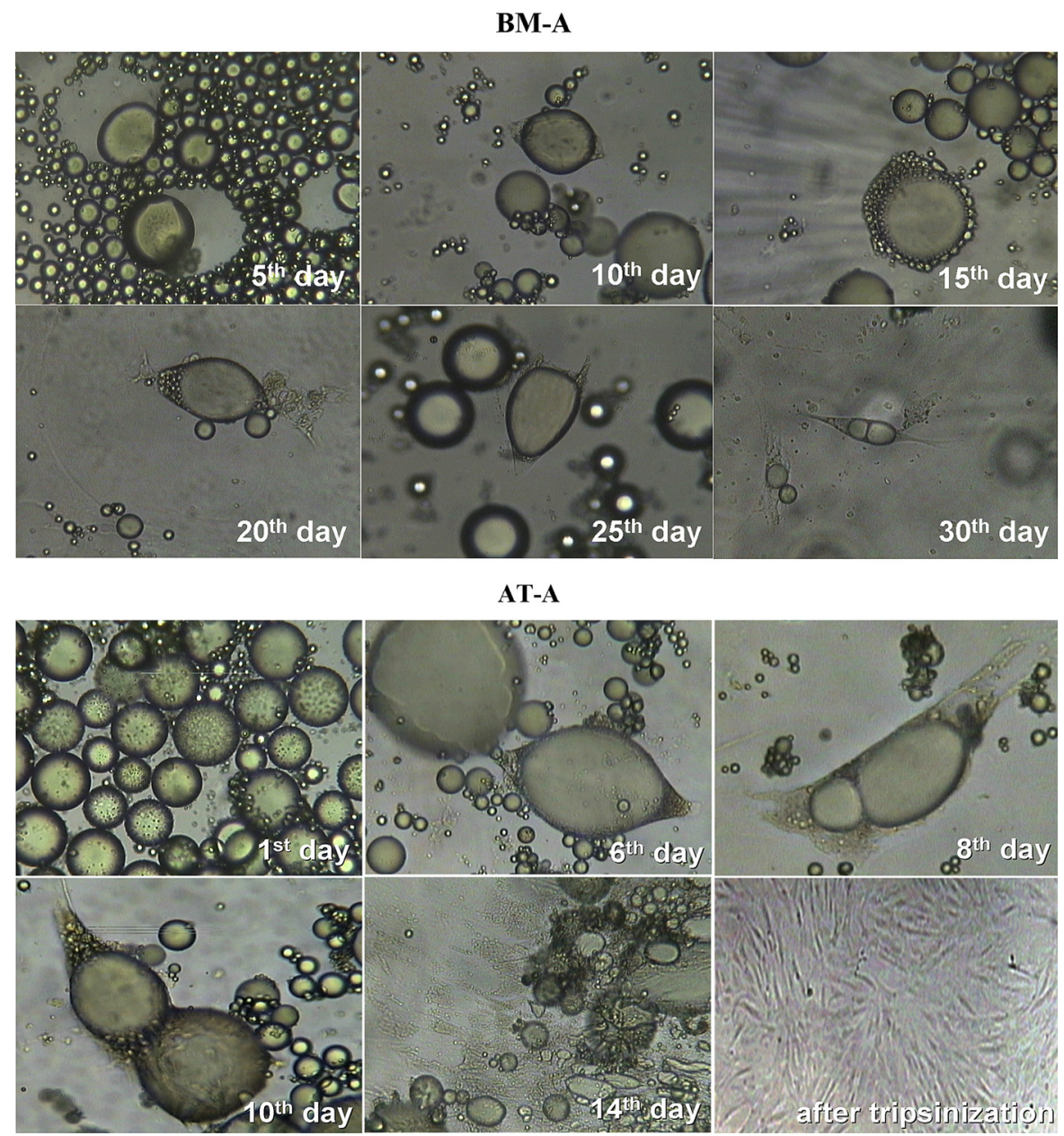

Figure 2. Liquid culture of BM-A and AT-A. During the culture time, only a few cells of BM-A samples changed their shape, whereas AT-A lost their intracytoplasmic lipid droplet and reached a fibroblast-like morphology.

Stem cells and reprogramming gene expression profile In a previous study [7], we showed the plastic properties of AT-A and their ability to differentiate into different cell lineages. AT-A expressed the four reprogramming genes Oct4, Klf4, c-myc, and Sox 2 and many stem cells genes required for self-renewal and pluripotency. To determine whether BM-A also have a stem cell-like phenotype, we analyzed the stem cell and cell reprogramming gene expression profile in BM-A and compared these results with AT-A. BM-A expressed some of the embryonic stem cell and cell reprogramming genes tested, with statistically significant differences from AT-A. Gata4, Tbx1, Sox17, Oct4, Klf4, and c-myc were expressed in both cell types; Nanog and Sox2 were not detectable in BMA (Fig. 4A). Moreover, quantitative data for cell reprogramming genes showed that Klf4 and c-myc were expressed in BM-A at lower levels compared with AT-A (Fig. 4B). These data suggest that mature BM fat expressed some important stem cell genes that are required for pluripotency.
Telomere length analysis

A terminal restriction fragment assay was performed for BM-A and AT-A using high telomere length as a stem cell feature. Data demonstrated that despite their mature properties, adipocytes have long telomeres. BM-A have long telomeres that are comparable with those of BMMSCs $(9.3 \pm 0.5$ vs. $9.7 \pm 0.3 \mathrm{~kb}$, respectively). AT-A showed telomere lengths longer than BM-A (11.5 \pm 0.7 $\mathrm{kb}$ ), and this result was in line with the great plasticity of AT-A previously demonstrated [7] (Fig. 5).

\section{Long-term culture-initiating cells}

To assess the function of BM-A in the BM microenvironment, we performed an LTC-IC assay. We evaluated the capacity of BM-A to support the survival and complete differentiation of hematopoietic stem cells by observing the CFU-GM and BFU-E at day 14 of culture. The total number of CFUs was $31 \pm 10$ in the presence of BM-A, $35 \pm 12$ in the presence of AT-A, and $40 \pm 16$ in the presence of AT-derived MSCs, which was used as a positive 
A
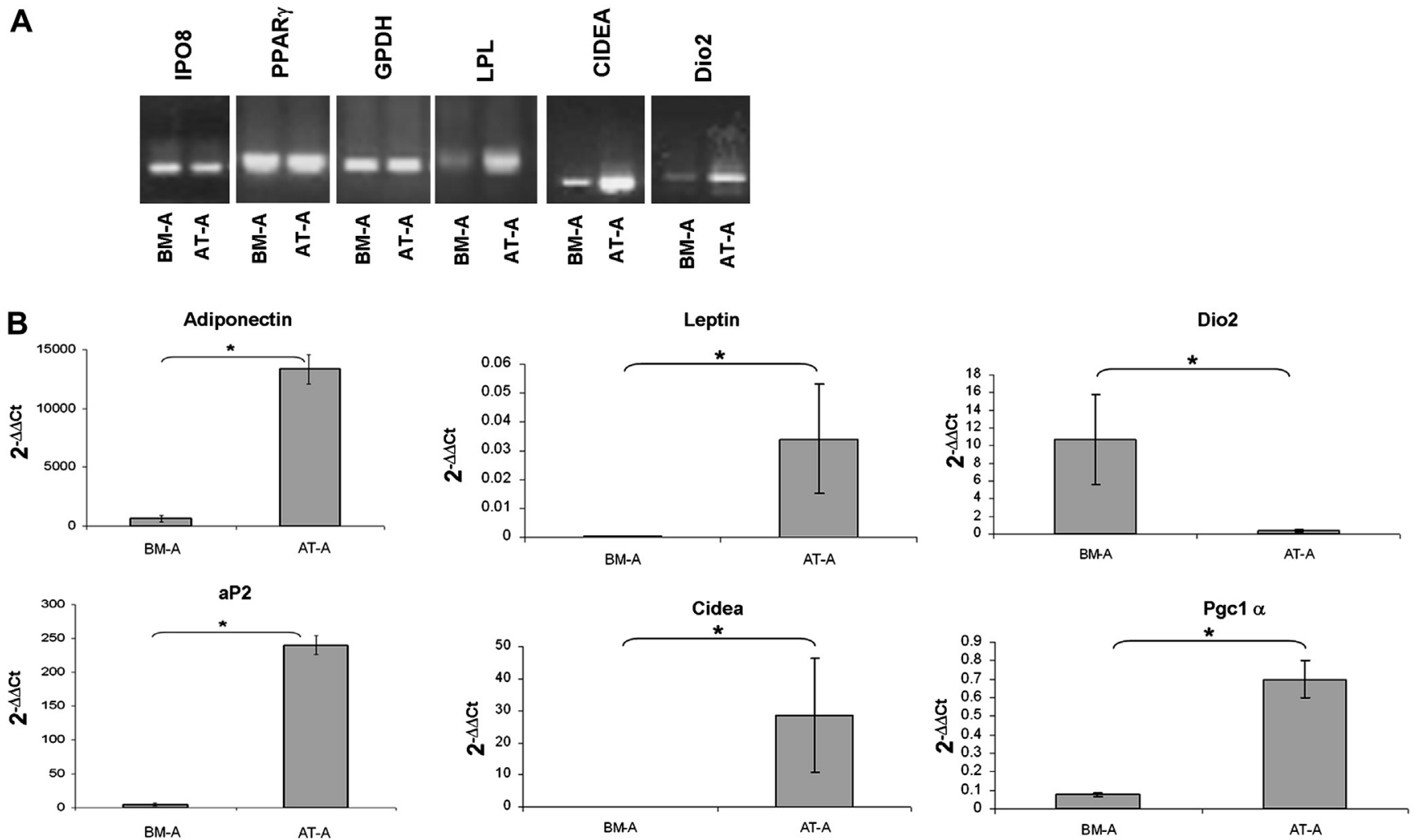

Figure 3. Molecular analysis for adipogenic lineage genes. (A) Qualitative expression of white and brown adipogenic markers in BM-A and AT-A. (B) Comparative expression level of Adipoq, aP2, Leptin, CIDEA, Dio2, and PGC1- $\alpha$ was performed by quantitative analysis in BM-A and AT-A. Data were analyzed using the $2^{-\Delta \Delta \mathrm{Ct}}$ method. Samples were run in duplicate, and the values are the mean $\pm \mathrm{SD}$. $* p<0.05$.

control group. No hematopoietic colonies were observed in the negative control. There was no significant difference in the number of CFUs between MSCs, which are known for their hematopoietic supporting capacity, and BM-A. Data suggested that BM-A have some properties of stromal cells with hematopoietic supporting function.

\section{Quantitative cytokines assay}

To investigate whether adipocytes support the proliferation of multipotent hematopoietic progenitors, we studied the amounts of stimulatory and inhibitory cytokines. The positive regulators of hematopoiesis, IL-6, IL-8, and G-CSF were secreted at high levels. GM-CSF was expressed at the same amount in BM-A and AT-A. Moreover, the negative regulators of hematopoietic microenvironment, IL-10, IL-17, MIP- $1 \alpha$ and TNF- $\alpha$, were not produced at significant levels in both cells tested (Supplementary Table E1, online only, available at www.exphem.org).

\section{Discussion}

Adipocytes are abundant cells in the human BM cavity and have not been studied extensively in this context. Indeed, the paucity of studies on this topic is most likely due to the difficulty in finding and isolating a sufficient number of $\mathrm{BM}$ adipocytes for experimentation. However, these cells represent a significant BM population that is implicated in metabolic function and a complex interrelationship with other stromal cells [12-14]. Previous observations [15] demonstrated that the fat content in the human BM increases with age, proceeding progressively from the periphery to the axial skeleton of the body. In this study, we analyzed human healthy BM-A from donors between 29 and 55 years old, despite the small number of cells in the young samples. We isolated a homogeneous population of BM-A with a typical white adipogenic morphology. BMA in BM samples showed a morphology (light and electron microscopy) similar to that described in other anatomic sites. Isolated and cultured BM-A showed a unilocular large cytoplasmic lipid droplet and a small flattened nucleus, maintaining their shape without noticeable lipolysis even after long-term culture. Moreover, the key transcriptional regulator of adipogenesis, PPAR $\gamma$, was expressed in BM$A$ in addition to some of its target genes, including GPDH, LPL, Adiponectin, aP2, and LEP. Krings et al. [16] showed that mouse marrow fat has a distinctive phenotype with respect to adipose tissue fat, which resembles both white and brown adipose tissue. In agreement with this study, we showed that BM-A expressed Dio2 and CIDEA, usually expressed in brown adipocytes, but we did not observe the expression of Prdm16 and notably of 
A

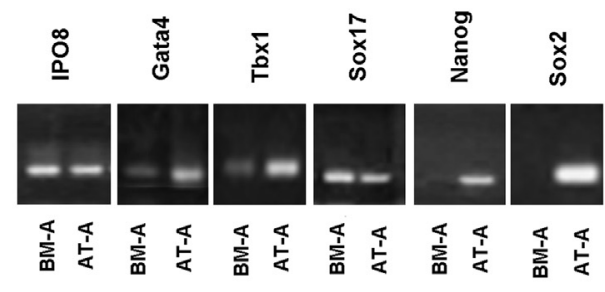

B
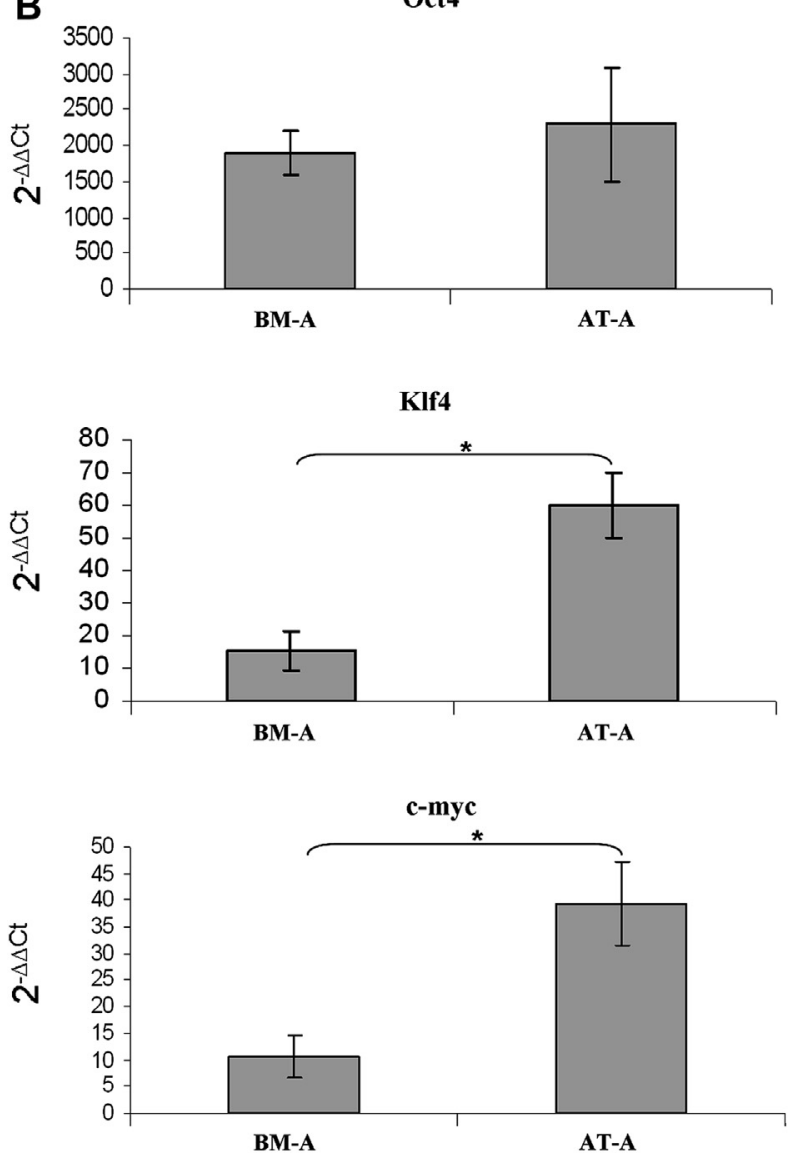

Figure 4. Molecular analysis for stem cell markers. (A) Qualitative expression of embryonic stem cell genes and cell-reprogramming genes was performed in BM-A and AT-A. (B) Quantitative expression of cellreprogramming genes was performed in BM-A and AT-A following the comparative $2^{-\Delta \Delta \mathrm{Ct}}$ method. Samples were run in duplicate, and the values are the mean $\pm \mathrm{SD} . * p<0.05$.

UCP1, the most important marker of brown adipocytes [17]. BM-A can provide a localized energy reservoir in the BM [18].

In this study, BM-A demonstrated to express stem cell markers. Indeed, the pattern of surface antigens of BM-A was similar to AT-A, suggesting the potential plasticity of these cells. Moreover, molecular data showed that BM-A expressed embryonic stem cell-like genes, including Gata4, Tbx1, and Sox17 [19], and reprogramming genes Oct4, Klf4, c-myc. Notably, BM-A did not express Sox2 and Nanog and possessed lower levels of Klf4 and c-myc with respect to AT-A. These results suggested that BM-A have more attenu-

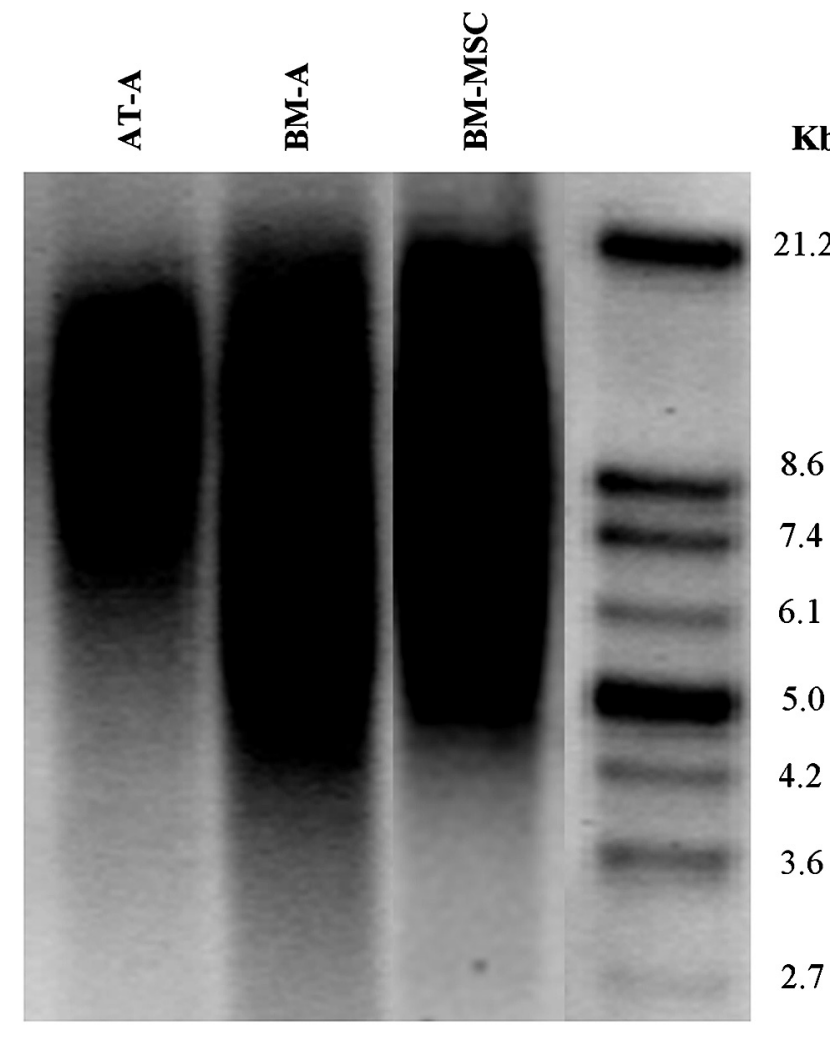

Figure 5. Telomere length analysis of BM-A. Southern blot analysis of the telomere length in BM-A, AT-A, and BM-derived MSCs. Results are representative of one independent experiment of BM, AT-A, and BMderived MSCs. BM-derived MSCs were used as positive controls.

ated plastic properties than the well-known plastic ability of AT-A [20]. Indeed, only a few cells from the BM-A samples were able to undergo lipolysis and revert into an MSC morphology. This failure of morphologic change was certainly linked to the expression of reprogramming genes. These data suggested that during the culture, the reprogramming genes were most likely important regulators of dedifferentiation and of the reprogramming process of mature adipocytes. In this study, we also analyzed BM-A telomere length as a stemness feature and compared these results with those for BM-MSCs. In a previous work $[11,21]$, we showed the results for fetal stem cells isolated from amniotic fluid and chorionic villi. These cells were a good source of MSCs, with telomeres of $13.4 \pm 0.3 \mathrm{~kb}$ and $12.30 \pm 1.03$ $\mathrm{kb}$, respectively. Data showed that BM-A have long telomeres $(9.3 \pm 0.5 \mathrm{~kb})$, similar to BM-MSCs $(9.7 \pm 0.3 \mathrm{~kb})$, thus suggesting again the potential plasticity of BM fat. These phenotypic and molecular data suggest that BM-A are mature and differentiated cells with some MSC properties such as stem cells genes expression, some surface antigens expression, and the telomere length. As a result, these cells cannot be defined as MSCs from a phenotypic and functional point of view, because they are not able to dedifferentiate, proliferate, and differentiate into mesenchymal lineages. 
Fat occupies a significant portion of BM cavity; however, its function is largely unknown. Therefore, the most important novelty of this work is the functional study of BM-A in the BM microenvironment. To study the role of BM-A in sustaining hematopoiesis, we analyzed the ability of these cells to maintain the survival and differentiation of hematopoietic stem cells. Several studies suggested that marrow fat might be involved in the metabolic support of hematopoiesis [22-24]. Bathija et al. [25] showed that BM-A are metabolically active cells that appear to be functionally related to hematopoiesis rather than to systemic fat storage. Laharrague et al. [24] demonstrated that BM-A could contribute to the hematopoiesis via the secretion of leptin in the vicinity of hematopoietic stem cells [26].

In this study, we have shown the interrelationship between mature BM-A and hematopoietic stem cells. Our data show that isolated mature adipocytes that were cocultured for a long period with $\mathrm{CD} 34^{+}$cells possess the ability to support the differentiation of hematopoietic stem cells. Moreover, our results show that BM-A are stromal cells with the same hematopoietic supporting function of ATderived MSCs and AT-A.

Corre et al. [27] used a similar coculture system of $\mathrm{CD} 34^{+}$cells seeded onto BM-derived adipocytes to investigate their role in supporting hematopoiesis. These differentiated cells supported complete myeloid and lymphoid differentiation from hematopoietic stem cells, but they did not support the proliferation of immature progenitors. Moreover, they showed that adipocytes that were differentiated from stromal vascular fraction cells secreted cytokines promoting the differentiation of committed hematopoietic progenitors, such as IL-6, G-CSF, and GMCSF, and cytokines that inhibit the proliferation of stem cells, such as MIP1- $\alpha$ [28]. In line with these observations, Naveiras et al. [29] demonstrated that mouse adipocyte-rich $\mathrm{BM}$ is associated with lower levels of hematopoietic progenitors. Moreover, Di Mascio et al. [30] demonstrated that adiponectin influences hematopoietic stem cells by increasing their proliferation while retaining the cells in a functionally immature state.

Our data showed that BM-A and AT-A expressed similar levels of some negative (IL-10, IL-17, MIP- $1 \alpha$, and TNF- $\alpha$ ) and positive regulators of hematopoiesis (IL-6, IL-8, GCSF, and GM-CSF). Interestingly, adipocytes secreted approximately the same amount of the cytokines tested as AT-derived MSCs, suggesting their capacity to support hematopoiesis.

\section{Conclusion}

Collectively, our results characterize BM-A at the morphologic, phenotypic, molecular, and functional levels. BM-A represents a population of white adipocytes with the same morphology of AT-A. The expression of typical white adipogenic markers and some regulatory genes of thermogenesis suggest that adipocytes do not have a passive role as space fillers in the BM, but instead play a role in metabolic activity through complex interrelationships with other stromal cells. BM-A expressed stem cell surface antigens and genes that are important for pluripotency and cell reprogramming. BM-A showed some important stemness features, such as the expression of some embryonic stem cell genes, as well as long telomeres, suggesting a possible plasticity of these cells. Moreover, BM-A are stromal cells with hematopoietic supporting capabilities that are most likely regulated by the production of specific molecules. Just for their stemness properties, the features and the role of BM-A could most likely change depending on the specific demands of the BM microenvironment.

\section{Acknowledgments}

This work was supported by grants from Associazione Italiana contro le leucemie, linfomi e mieloma, sezione di Ancona-ONLUS and EU FP7 project Diabet (Health-F2-2011- 278373).

\section{Conflict of interest disclosure}

No financial interest/relationships with financial interest relating to the topic of this article have been declared.

\section{References}

1. Lecka-Czernik B. Marrow fat metabolism is linked to the systemic energy metabolism. Bone. 2012;50:534-539.

2. Tavassoli M, ed. Handbook of the hematopoietic microenvirnment. Clifton, NJ: Humana Press; 1989.

3. Calvo W, Fliedner TM, Herbst E, Hügl E, Bruch C. Regeneration of bloodforming organs after autologous leukocyte transfusion in lethally irradiated dogs. II. Distribution and cellularity of the marrow in irradiated and transfused animals. Blood. 1976;47: 593-601.

4. Cinti S. Transdifferentiation properties of adipocytes in the adipose organ. Am J Physiol Endocrinol Metab. 2009;297:E977-E986.

5. De Matteis R, Zingaretti MC, Murano I, et al. In vivo physiological transdifferentiation of adult adipose cells. Stem Cells. 2009;27: 2761-2768.

6. Smorlesi A, Frontini A, Giordano A, Cinti S. The adipose organ: white-brown adipocytes plasticity and metabolic inflammation. Obes Rev. 2012;13(suppl 2):83-96.

7. Poloni A, Maurizi G, Leoni P, et al. Human dedifferentiated adipocytes show similar properties to bone marrow-derived mesenchymal stem cells. Stem Cells. 2012;30:965-974.

8. Morroni M, Giordano A, Zingaretti MC, et al. Reversible transdifferentiation of secretory epithelial cells into adipocytes in the mammary gland. Proc Natl Acad Sci U S A. 2004;101:16801-16806.

9. Zhang HH, Kumar S, Barnett AH, Eggo MC. Ceiling culture of mature human adipocytes: use in studies of adipocyte functions. J Endocrinol. 2000;164:119-128.

10. Livak KJ, Schmittgen TD. Analysis of relative gene expression data using real-time quantitative PCR and the $2^{-\Delta \Delta \mathrm{Ct}}$ method. Methods. 2001;25:402-408.

11. Poloni A, Maurizi G, Babini L, et al. Human mesenchymal stem cells from chorionic villi and amniotic fluid are not susceptible to transformation after extensive in vitro expansion. Cell Transplant. 2011;20: $643-654$ 
12. Rosen CJ, Ackert-Bicknell C, Rodriguez JP, Pino AM. Marrow fat and the bone microenvironment: developmental, functional, and pathological implications. Crit Rev Eukaryot Gene Expr. 2009;19:109-124.

13. Gimble JM, Nuttall ME. Bone and fat: old questions, new insights. Endocrine. 2004;23:183-188.

14. Gimble JM, Zvonic S, Floyd ZE, Kassem M, Nuttall ME. Playing with bone and fat. J Cell Biochem. 2006;98(2):251-266.

15. Tuljapurkar SR, McGuire TR, Brusnahan SK, et al. Changes in human bone marrow fat content associated with changes in hematopoietic stem cell numbers and cytokine levels with aging. J Anat. 2011;219:574-581.

16. Krings A, Rahman S, Huang S, Lu Y, Czernik PJ, Lecka-Czernik B. Bone marrow fat has brown adipose tissue characteristics, which are attenuated with aging and diabetes. Bone. 2012;50:546-552.

17. Cannon B, Nedergaard J. Brown adipose tissue: function and physiological significance. Physiol Rev. 2004;84:277-359.

18. Gimble JM, Robinson CE, Wu X, Kelly KA. The function of adipocytes in the bone marrow stroma: an update. Bone. 1996;19:421-428.

19. Cai J, Li W, Su H, et al. Generation of human induced pluripotent stem cells from umbilical cord matrix and amniotic membrane mesenchymal cells. J Biol Chem. 2010;285:11227-11234.

20. Cinti S. The adipose organ at a glance. Dis Model Mech. 2012;5: 588-594.

21. Poloni A, Maurizi G, Serrani F, et al. Human AB serum for generation of mesenchymal stem cells from human chorionic villi: comparison with other source and other media including platelet lysate. Cell Prolif. 2012;45:66-75.
22. Bathija A, Davis S, Trubowitz S. Marrow adipose tissue: response to erythropoiesis. Am J Hematol. 1978;5:315-321.

23. Gainsford T, Willson TA, Metcalf D, et al. Leptin can induce proliferation, differentiation and functional activation of hemopoietic cells. Proc Natl Acad Sci U S A. 1996;93:14564-14568.

24. Laharrague P, Larrouy D, Fontanilles AM, et al. High expression of leptin by human bone marrow adipocytes in primary culture. FASEB J. 1998;12:747-752.

25. Bathija A, Davis S, Trubowitz S. Bone marrow adipose tissue: response to acute starvation. Am J Hematol. 1979;6:191-198.

26. Laharrague P, Truel N, Fontanilles AM, et al. Regulation by cytokines of leptin expression in human bone marrow adipocytes. Horm Metab Res. 2000;32:381-385.

27. Corre J, Planat-Benard V, Corberand JX, Pénicaud L, Casteilla L, Laharrague P. Human bone marrow adipocytes support complete myeloid and lymphoid differentiation from human CD34 cells. Br J Haematol. 2004; 127:344-347.

28. Corre J, Barreau C, Cousin B, et al. Human subcutaneous adipose cells support complete differentiation but not self-renewal of hematopoietic progenitors. J Cell Physiol. 2006;208:282-288.

29. Naveiras O, Nardi V, Wenzel PL, Hauschka PV, Fahey F, Daley GQ. Bone marrow adipocytes as negative regulators of the haematopoietic microenvironment. Nature. 2009;460:259-263.

30. DiMascio L, Voermans C, Uqoezwa M, et al. Identification of adiponectin as a novel hemopoietic stem cell growth factor. J Immunol. 2007; 178:3511-3520 

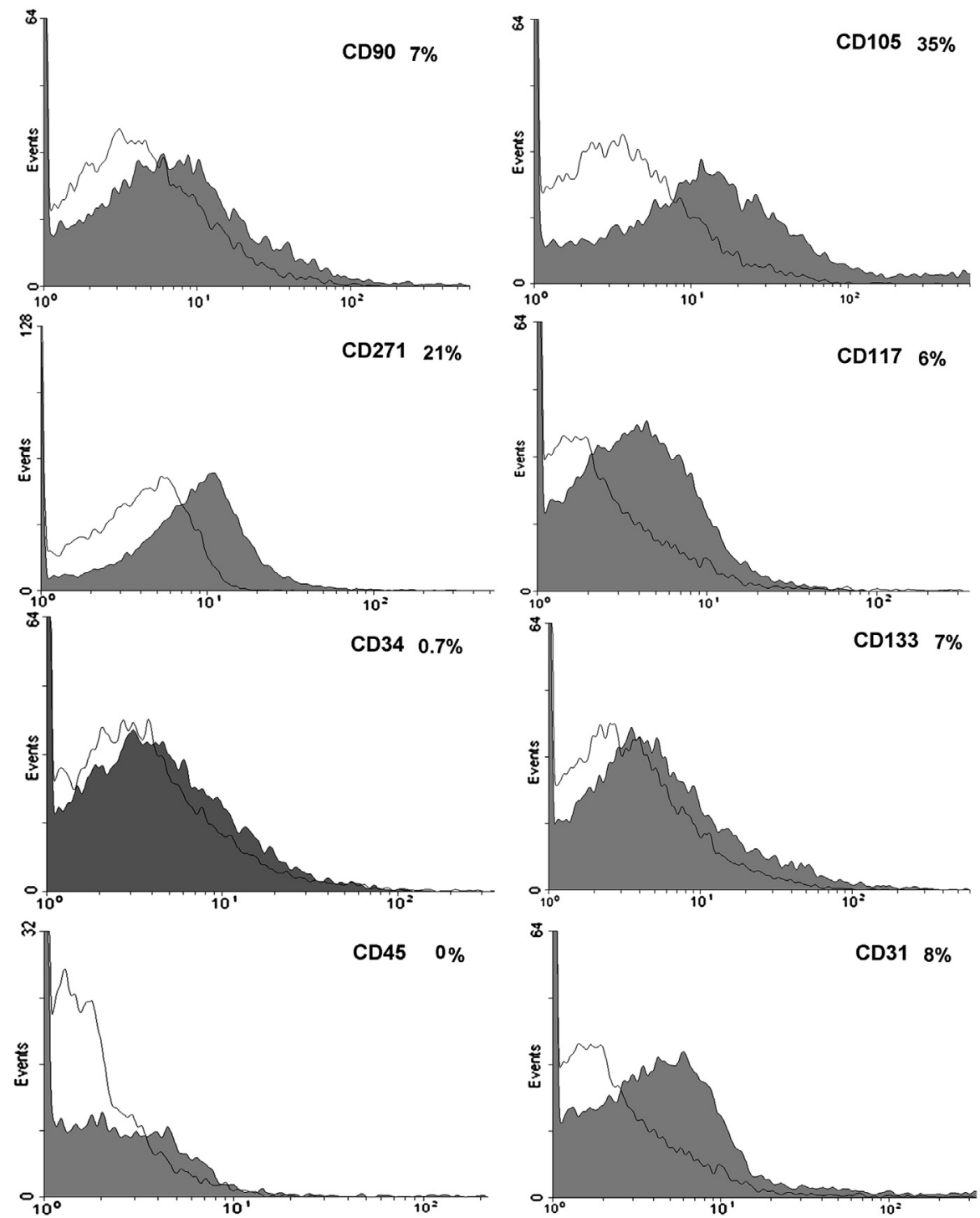

Supplementary Figure E1. Immunophenotypic analysis of BM-A. FACS analysis of surface antigen markers on BM-A. The open histograms indicate negative controls. Results are representative of one independent experiment of isolated BMA. 
Supplementary Table E1. Quantitative cytokines assay: levels of factors secreted from BM-A and AT-A

\begin{tabular}{lccc}
\hline Cytokines & BM-A & AT-A & SVF-MSC \\
\hline IL-6 & + & + & ++ \\
IL-8 & ++ & ++ & ++ \\
IL-10 & - & - & - \\
IL-17 & - & - & - \\
G-CSF & + & + & + \\
GM-CSF & - & - & + \\
MIP-1 $\alpha$ & - & - & - \\
TNF- $\alpha$ & - & - & + \\
\hline
\end{tabular}

AT- $\mathrm{A}=$ adipocytes derived from adipose tissue; $\mathrm{BM}-\mathrm{A}=$ adipocytes isolated from human bone marrow; G-CSF = granulocyte colony-stimulating factor; GM-CSF = granulocyte-macrophage colony-stimulating factor; MIP = macrophage inflammatory protein; SVF-MSC $=$ stromal vascular fraction-derived mesenchymal stem cells; $\mathrm{TNF}=$ tumor necrosis factor. Results from the averages of four samples are shown:,$-<50 \mathrm{pg} / \mathrm{mL} ;+$, 50 to $<500 \mathrm{pg} / \mathrm{mL} ;++, 500$ to $<5000 \mathrm{pg} / \mathrm{mL} ;+++,>5000 \mathrm{pg} / \mathrm{mL}$.

There were no significant differences between the cells analyzed. 